\title{
Detection of interaction-induced nonlocal effects using perfectly transmitting nanostructures
}

\author{
Dietmar Weinmann ${ }^{1}$, Rodolfo A. Jalabert ${ }^{1}$, Axel Freyn ${ }^{2}$, Gert-Ludwig Ingold ${ }^{3}$ and Jean-Louis \\ PICHARD ${ }^{2}$ \\ 1 Institut de Physique et Chimie des Matériaux de Strasbourg, UMR 7504 (CNRS-ULP) - 23 rue du Loess, BP 43, \\ F-67034 Strasbourg Cedex 2, France \\ ' 2 Service de Physique de l'État Condensé (CNRS URA 2464), DSM/IRAMIS/SPEC, CEA Saclay - F-91191 Gif sur \\ Yvette Cedex, France \\ 3 Institut für Physik, Universität Augsburg - D-86135 Augsburg, Germany
}

PACS 73.23.-b - Electronic transport in mesoscopic systems

PACS 71.10.- - - Theories and models of many-electron systems

PACS 73.63.Nm - Quantum wires

\begin{abstract}
We consider one-dimensional transport through an interacting region in series with a point-like one-body scatterer. When the conductance of the interacting region is perfect, independently of the interaction strength, a nonlocal interaction effect yields a total conductance of the composed system that depends on the interaction strength and is lower than the transmission of the one-body scatterer. This qualitative nonlocal effect allows to probe the dressing cloud of an interacting system by ideal noninteracting leads. The conductance correction increases with the strength of the interaction and the reflection of the one-body scatterer (attaining relative changes $>50 \%$ ), and decreases with the distance between the interacting region and the one-body scatterer. Scaling laws are obtained and possible experimental realizations are suggested.
\end{abstract}

Introduction. - Viewing quantum transport as a scattering problem is at the heart of Landauer's approach to the conductance of mesoscopic systems [1]. Working at zero temperature and ignoring electron-electron interactions we solely have to consider the elastic scattering ' of electrons at an energy that is given by the Fermi level of the electrodes, arising from one-body potentials in the system. Including interactions through Landau quasiparticles does not modify appreciably this situation. Such ' an effective one-body description allows to understand a wealth of phenomena, ranging from residual resistivity to interference effects.

Interaction effects become prominent in small and weakly connected quantum dots displaying Coulomb blockade oscillations [1]. Describing the charging effects through capacitances one stays at the level of a simplified local mean-field approach, and the view of quantum transport as the scattering of (quasi-)particles is still applicable. Exploring yet smaller systems within the hypothesis of an effective one-body scattering we encounter cases in which the effective transmission can no longer be obtained from such a simple approach ignoring many-body exchange and correlation effects. Such many-body signatures have been theoretically demonstrated for the 0.7 anomaly of quantum point contacts [2], in the length-dependent oscillations of the conductance through an atomic chain [3], and in the interaction-induced increase of the conductance through a strongly disordered quantum wire [4]. In realistic systems it is difficult to control the effective interaction strength independently of other parameters. Thus, the clear-cut observation of many-body signatures on the measured transmissions has remained elusive.

For systems containing a small region, that we refer to as nanosystem and in which interactions are important, the situation is even more complicated since one can pose the fundamental question under which circumstances the one-body scattering approach yielding an effective transmission is valid. Kondo physics in the transport through ultra-small quantum dots $[5,6]$ provides an example where electronic correlations are necessary for the interpretation of the data and where one-body concepts cannot be used inside the spin screening cloud $[7,8]$.

In generic nanosystems, the effective one-body approaches are challenged by the nonlocal effects arising 
from interactions which can be tested by approaching an external scatterer. The nonlocality can be explained already at the Hartree-Fock (HF) level [9-11], since the Hartree and Fock corrections are given by nonlocal coupled integral equations. For instance, the effect of an external scatterer upon the Hartree corrections results from the Friedel oscillations of the electron density that the external scatterer induces inside the nanosystem. The Fock corrections are characterized by similar oscillations [9]. Using a model where particle-hole symmetry yields a uniform density, the nonlocal effect arises from the exchange. At zero temperature, introducing an external scatterer at a distance $L_{\mathrm{C}}$ of the nanosystem yields an effect which decays as the Friedel oscillations which cause it, i.e. $\propto 1 / L_{\mathrm{C}}$ in one dimension. At a finite temperature $T$, this effect is exponentially suppressed [9], if $L_{\mathrm{C}}$ exceeds the thermal length $L_{\mathrm{T}}$ describing the scale over which the electrons at the Fermi surface propagate during a time $\hbar / k_{B} T$. This means that all the external scatterers located in a region of size $L_{\mathrm{T}}$ can modify the transmission of the interacting nanosystem.

Only the dressed nanosystem, consisting of the interacting region and its local environment, behaves as an effective one-body scatterer. The fundamental question posed above translates into the question about the nature and the extension of the associated cloud dressing the nanosystem. A way of testing such a cloud is to study the conductance through two nanosystems connected in series by a short noninteracting lead, and to detect the deviations from the prediction based on the combination of effective one-body scatterers [12]. This is in principle an experimentally observable effect, although it seems difficult to get simultaneous access to both, the conductance of the individual and of the combined system, and/or to change the separation between the interacting regions.

Within this line of investigations it seems more promising to replace one of the nanosystems by a tunable onebody scatterer. If the one-body scatterer is influenced by an attached Aharonov-Bohm ring, a HF treatment indicates that a nonlocal interaction effect can lead to a significant dependence of the nanosystem transmission on the magnetic flux piercing the ring $[10,11]$. If the one-body scatterer is a scanning gate microscope acting on a twodimensional electron gas in the proximity of a quantum point contact $[13,14]$, its effect upon the resulting conductance carries the signature of the electron-electron interactions inside the constriction [15]. In these two cases the interaction determines both, the effective transmission of the nanosystem as well as the interaction-induced nonlocal correction to the total conductance. However, if the conductance of the nanosystem is independent of the interaction strength, the only source of interaction dependence of the total conductance can be the nonlocal interaction effect. This is a striking situation because an interaction-dependent conductance implies the nonapplicability of the standard composition law and therefore immediately demonstrates the presence of nonlocal effects.

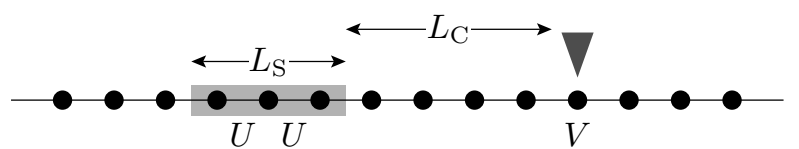

Fig. 1: One-dimensional setup with an interacting region (nanosystem, grey) of length $L_{\mathrm{S}}$ and a local one-body scattering potential $V$ at a distance $L_{\mathrm{C}}$.

In this work we consider precisely this situation by setting up a one-dimensional model with parameters chosen such that the transmission through the nanosystem is perfect for all values of the interaction strength [3,4], while the one-body point-like scatterer is introduced as an electrostatic perturbation.

Nanosystem with perfect conductance. - We consider spinless fermions in a one-dimensional chain with an interacting region of length $L_{\mathrm{S}}$, separated by a lead of length $L_{\mathrm{C}}$ from a point-like scatterer (see Fig. 1). The corresponding Hamiltonian reads

$$
H=H_{\text {kin }}+H_{\text {int }}+H_{1 \mathrm{bs}},
$$

where

$$
H_{\text {kin }}=-\sum_{i=-\infty}^{\infty}\left(c_{i}^{\dagger} c_{i+1}+\text { h.c. }\right)
$$

is the kinetic energy part. Here, $c_{i}$ annihilates a fermion on site $i$, and we have fixed the energy scale by setting the hopping amplitude equal to unity. The nearest-neighbour interaction on sites 1 to $L_{\mathrm{S}}$ is described by

$$
H_{\mathrm{int}}=U \sum_{i=1}^{L_{\mathrm{S}}-1}\left(n_{i}-1 / 2\right)\left(n_{i+1}-1 / 2\right)
$$

with the local density operators $n_{i}=c_{i}^{\dagger} c_{i}$.

At half filling, an odd number of sites $L_{\mathrm{S}}$ ensures a perfect effective transmission, i.e. a dimensionless conductance $g=G /\left(e^{2} / h\right)=1$, for the nanosystem, independent of the interaction strength $[3,4,16]$. We choose half filling and $L_{\mathrm{S}}=3$ in order to keep the size of the total system as small as possible.

The one-body on-site scattering potential of height $V$ that is separated from the interacting region by $L_{\mathrm{C}}$ sites is represented by

$$
H_{1 \mathrm{bs}}=V n_{L_{\mathrm{S}}+L_{\mathrm{C}}+1} .
$$

It is straightforward to calculate the transmission probability and thus the conductance $g_{1 \mathrm{bs}}$ of an on-site potential of strength $V$ in a clean chain. At energy $E=0$ corresponding to the Fermi energy at half filling, one gets

$$
g_{1 \mathrm{bs}}=\frac{4}{4+V^{2}} .
$$

For two one-body scatterers in series, where the first one is characterized by perfect transmission, the total transmission is simply given by the transmission of the second 


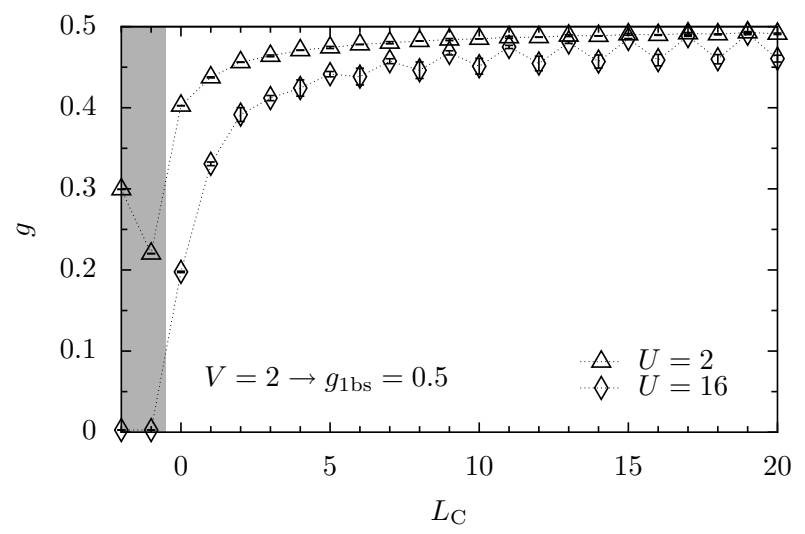

Fig. 2: Conductance of the combined system sketched in Fig. 1 as a function of $L_{\mathrm{C}}$ for a scatterer with $V=2$ for $U=2$ (triangles) and $U=16$ (diamonds). The grey area indicates that the scatterer is placed in the interacting region.

scatterer. In our case, one would therefore naively expect a total conductance $g=g_{1 \mathrm{bs}}$. However, the perfect transmission of the interacting region is an effective transmission describing the interacting region including long attached leads. The presence of a one-body scatterer in the vicinity of the nanosystem affects its transmission. We will show that this leads to pronounced deviations of the total conductance from $g_{1 \mathrm{bs}}$.

The embedding method $[2,4,16-19]$ allows to calculate the zero-temperature linear conductance through the system composed of the interacting part and the one-body scatterer in series. Within this method the conductance is extracted from the charge stiffness of a ring composed by the system and a long noninteracting lead in the limit of infinite lead length. We use the DMRG algorithm $[20,21]$ to determine the stiffness $D$ for different ring sizes $L$ up to 120 sites and extrapolate the results to infinite system size using fits of second-order polynomials to the numerical data for $\log D$ as a function of $1 / L$. An estimate for the precision of the resulting extrapolated value is given by the difference of the result as compared to the one obtained from a linear extrapolation of $\log D(1 / L)$. The resulting precision of the extracted conductance is displayed by the error bars in some of the figures. Using the DMRG algorithm allows to obtain exact results for finite system sizes in contrast to the HF approximation, which fails when $U$ or $L_{\mathrm{S}}$ become large and induce nonnegligible correlation effects [9].

The dependence of the conductance $g$ on the length $L_{\mathrm{C}}$ for an on-site potential $V=2$ is shown in Fig. 2 2 for two different values of the interaction strength. In this case, the conductance of the one-body scatterer is $g_{1 \mathrm{bs}}=0.5$. Pronounced deviations from that value appear at small separations between the scatterer and the nanosystem. Those deviations are negative and increase with decreasing $L_{\mathrm{C}}$. While this seems reminiscent of the increase of the deviations observed for even values of $L_{\mathrm{C}}$ when two nanosystems in series are considered [12], there is nevertheless an

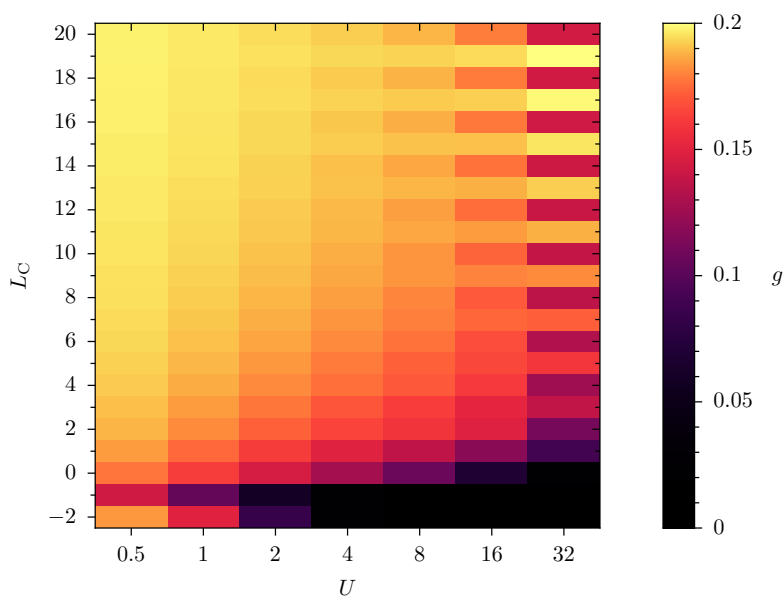

Fig. 3: Conductance $g$ as a function of $L_{\mathrm{C}}$ and $U$ for a scatterer with strength $V=4$ corresponding to $g_{1 \mathrm{bs}}=0.2$.

important difference. In the present case, deviations from $g_{1 \text { bs }}$ appear for all values of $L_{\mathrm{C}}$. For not too strong interaction strength $U=2$, the deviations increase to $20 \%$ of $g_{1 \mathrm{bs}}$ as $L_{\mathrm{C}}$ decreases down to zero. For $U=16$, the effect is stronger $\left(60 \%\right.$ of $\left.g_{1 \mathrm{bs}}\right)$ and even-odd oscillations as a function of $L_{\mathrm{C}}$ appear for large values of $L_{\mathrm{C}}$. The negative values of $L_{\mathrm{C}}$ indicated in grey correspond to the case where the potential scatterer is located inside the nanosystem. The deviation from the noninteracting conductance is particularly strong for $L_{\mathrm{C}}=-1$, when the one-body scatterer acts at the edge of the three interacting sites.

The behaviour of the total conductance $g$ as a function of $U$ and $L_{\mathrm{C}}$ is shown in Fig. 3 for $V=4$, that corresponds to a conductance of $g_{1 \mathrm{bs}}=0.2$ for the one-body scatterer alone. The total conductance is equal to $g_{1 \mathrm{bs}}$ in the noninteracting case $(U=0)$ and starts to decrease with increasing $U$. In addition, it can be seen that the oscillations of $g$ with $L_{\mathrm{C}}$ appear already for moderate interaction strength. These oscillations and the deviations of the total conductance $g$ from $g_{1 \text { bs }}$ become stronger as $U$ increases. As a consequence, $g$ assumes large values approaching $g_{1 \mathrm{bs}}$ for large $U$ when $L_{\mathrm{C}}$ is odd.

The $U$-dependence of the deviations of $g$ from $g_{1 \mathrm{bs}}$ contrasts with the situation for two interacting regions in series [12] where the deviations from the noninteracting composition law of scatterers reach a maximum around $U=2$ and decrease for stronger values of $U$. The qualitative difference in the behaviour arises from the fact that in the case considered in Ref. [12], for even $L_{\mathrm{C}}$ the conductance of the interacting nanosystems and the total conductance decrease with increasing interaction strength. This results in a decrease of the nonlocal corrections, which are further reduced by the effective decoupling of the nanosystem from the noninteracting leads occurring in the limit of strong interactions [22].

Apart from the oscillations which are particularly noticeable at strong interaction, the decrease of the nonlocal 


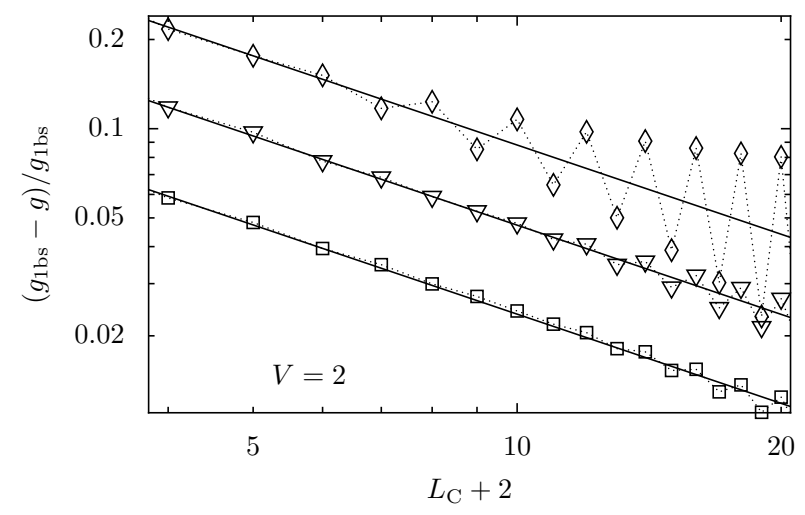

Fig. 4: Log-log plot of the relative conductance correction as a function of $L_{\mathrm{C}}+2$ at $V=2$, for $U=1$ (squares), $U=4$ (triangles), and $U=16$ (diamonds). The full lines are fits of (6) while the dotted lines serve to guide the eye.

correction with $L_{\mathrm{C}}$ is quite well described by the scaling

$$
\frac{g_{1 \mathrm{bs}}-g}{g_{1 \mathrm{bs}}}=\frac{A(U, V)}{L_{\mathrm{C}}+2} \text {. }
$$

This is shown in Fig. 4 and confirms the expected scaling due to Friedel oscillations caused by the one-body scatterer influencing the nanosystem whose centre is at a distance $L_{\mathrm{C}}+2$ from the perturbing tip.

Potential scatterer versus weak link. - The potential scatterer studied so far breaks particle-hole symmetry, and therefore disturbs the uniform electron density at half filling in the perfectly transmitting nanosystem. It is then important to study whether the nonlocal effect changes when the one-body scatterer does not break particle-hole symmetry. This can be achieved by using a weak link as a one-body scatterer, where the term $H_{1 \mathrm{bs}}$ in the Hamiltonian (11) is set to

$$
H_{1 \mathrm{bs}}=\left(1-t_{\mathrm{wl}}\right)\left(c_{L_{\mathrm{S}}+L_{\mathrm{C}}+1}^{\dagger} c_{L_{\mathrm{S}}+L_{\mathrm{C}}}+\text { h.c. }\right)
$$

replacing the hopping matrix element for the link between the sites $L_{\mathrm{S}}+L_{\mathrm{C}}$ and $L_{\mathrm{S}}+L_{\mathrm{C}}+1$ by $t_{\mathrm{wl}}$. For the conductance of the weak link alone at the Fermi energy one obtains

$$
g_{1 \mathrm{bs}}=\frac{4}{\left(t_{\mathrm{wl}}+1 / t_{\mathrm{wl}}\right)^{2}} .
$$

The results for the conductance of the combined system are very similar to the situation of a potential scatterer in series with an interacting region. In Fig. 5 we compare the relative change of the conductance due to the interactions as a function of the uncorrelated conductance $g_{1 \text { bs }}$ of the one-body scatterer alone, for $L_{\mathrm{C}}=3$. We plot the dependence of the relative change $\left(g_{1 \mathrm{bs}}-g\right) / g_{1 \mathrm{bs}}$ of the total conductance on the conductance $g_{1 \mathrm{bs}}$. The full symbols are data points obtained for a potential scatterer described by (4) while the open symbols stand for a system where the one-body scatterer is modeled as a weak

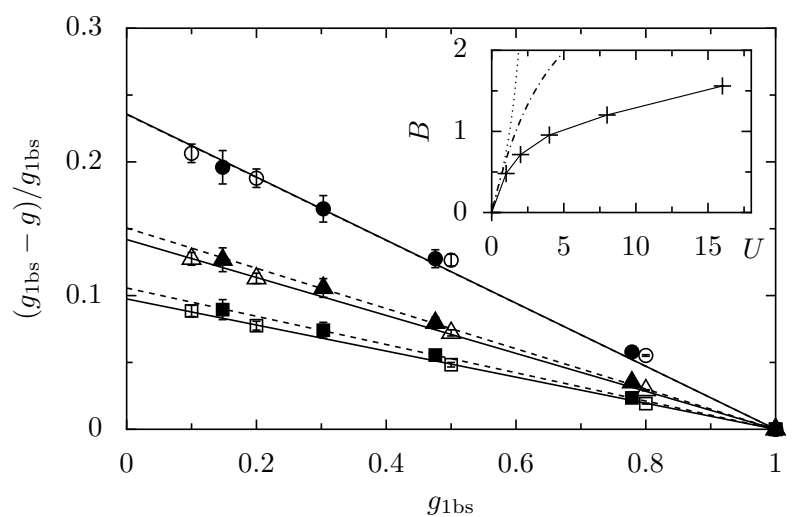

Fig. 5: Relative change of the conductance as a function of the conductance of the one-body scatterer arising from an on-site potential (empty symbols; $V=1,2,4$, and 6 ) and a weak link (filled symbols; $t_{\mathrm{wl}}=0.2,0.3,0.4$, and 0.6), at $L_{\mathrm{C}}=3$. Squares, triangles and circles represent data for $U=1,2$, and 8, respectively. Linear fits of the form (9) are shown for an on-site potential and a weak link as solid and dashed lines, respectively. Inset: Parameter $B$ of the fit (9) for the case of a potential scatterer as a function of $U$ for $L_{\mathrm{C}}=1$. The results for $L_{\mathrm{C}}=3$ and 5 as well as for a weak link collapse on the same line with deviations below $3.2 \%$. HF results at $L_{\mathrm{C}}=1$ for the slope at $g_{1 \mathrm{bs}}=1$ are shown for a potential scatterer (dotted line) and a weak link (dashed-dotted line).

link (77). The solid and dashed lines are the corresponding linear fits. The two different ways of modeling the onebody scatterer yield nonlocal effects that are very close, demonstrating that the nonlocal correlation effect scales with the conductance $g_{1 \mathrm{bs}}$, independent of the nature of the one-body scatterer. As expected, the relative change of the conductance due to the interaction effect increases monotonically with decreasing $g_{1 \mathrm{bs}}$.

Already for $L_{\mathrm{C}}=3$ the nonlocal effect can amount to a conductance change of more than $20 \%$. This value increases beyond $60 \%$ for $L_{\mathrm{C}}=0$, when the on-site potential is applied on the first noninteracting site.

For not too strong interaction, the dependence follows approximately the linear relationship

$$
\frac{g_{1 \mathrm{bs}}-g}{g_{1 \mathrm{bs}}}=B(U) \frac{1-g_{1 \mathrm{bs}}}{L_{\mathrm{C}}+2},
$$

yielding $A\left(U, g_{1 \mathrm{bs}}\right)=B(U)\left(1-g_{1 \mathrm{bs}}\right)$ for the parameter $A$ in (6). For the whole range of explored parameters, the $U$-dependence of $B$, shown in the inset of Fig. [5 is monotonically increasing. The collapse of the data on a universal curve confirms the scaling (6).

The scaling law (9) represents evidence for an intrinsic property of the cloud dressing the nanosystem, namely that an external scatterer placed in the proximity of the perfectly transmitting nanosystem yields a universal renormalized conductance $g$ given by (9).

A comparison of the quasi-exact results obtained using DMRG to HF results (grey lines) is shown in the inset of Fig. 5. As it turns out that the linearity of the scaling (9) 


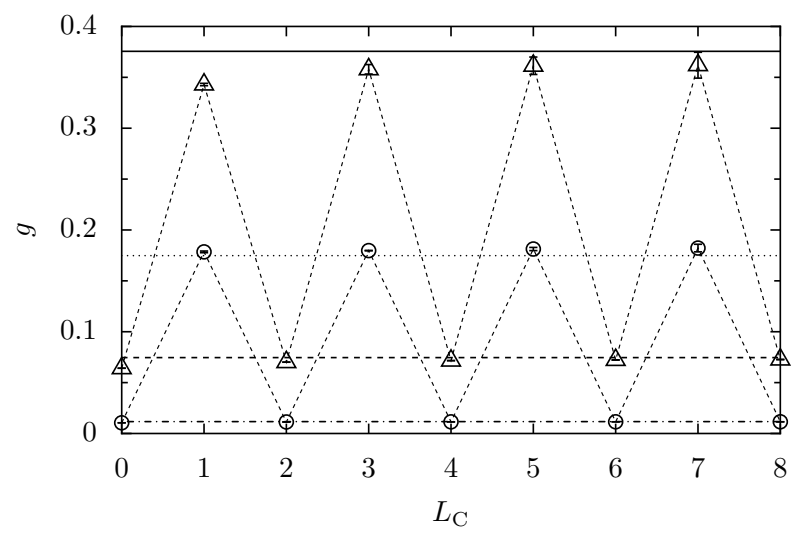

Fig. 6: Conductance $g$ as a function of $L_{\mathrm{C}}$ for an interacting region of length $L_{\mathrm{S}}=2$ and a potential scatterer with $V=4$. Triangles and circles are for $U=2$ and 8 , respectively. The horizontal lines are the predictions using the noninteracting composition law of scatterers.

is not satisfied by the HF results, the parameter $B$ is deduced from the slope at $g_{1 \mathrm{bs}}=1$. The linear scaling of the nonlocal conductance correction with $g_{1 \mathrm{bs}}$, independent of the nature of the scatterer, persists in the quasi-exact results beyond $U \simeq 10$, while $\mathrm{HF}$ yields different results for different scatterers having the same $g_{1 \mathrm{bs}}$. Since the HF results exhibit the universality of the exact results only at rather weak interaction, the cloud dressing the nanosystem carries the signature of electronic correlations.

Nanosystem with interaction-dependent conductance. - The results presented above show that the nonlocal effects in the conductance appear in a particularly spectacular fashion when the nanosystem has perfect transmission. When the transmission through the nanosystem is interaction-dependent, the signature of nonlocal interaction effects has to be extracted from the difference between the total conductance and the prediction resulting from the composition of the effective interactiondependent scatterer corresponding to the nanosystem with the one-body scatterer. Fig. 6 presents the case where the electrons interact only inside a nanosystem of length $L_{\mathrm{S}}=$ 2 , such that the effective transmission of the nanosystem depends on $U[3,4]$. The total conductance exhibits strong even-odd oscillations as a function of $L_{\mathrm{C}}$ that are most pronounced when the values of the transmissions of the onebody scatterer and the nanosystem are close, such that the noninteracting composition law predicts Fabry-Pérotlike oscillations. The deviations of the total conductance from the noninteracting composition law represented by the horizontal lines are much smaller than for the case of a perfectly transmitting interacting nanosystem. This example shows that choosing a nanosystem with perfect conductance allows for a qualitative effect of nonlocal interactions, unlike the merely quantitative corrections in the general case.
Discussion. - We have demonstrated that a nanosystem connected in series with a one-body scatterer constitutes an ideal configuration to identify the nonlocality of the transmission in the presence of interaction and to detect how it is dressed by the attached leads. In particular, when the transmission through the nanosystem is perfect, the nonlocal interaction effects can be unambiguously identified because they result in dependencies of the conductance of the total system on the interaction strength and on the position of the one-body scatterer.

The experimental confirmation of this striking effect necessitates a perfectly transmitting nanostructure together with the ability to control the interaction strength and/or the distance between the nanostructure and the one-body scatterer. Silicon quantum wires with nanosize MOSFETs allow to define regions with strong local enhancement of the effective electron-electron interaction [23]. However, considerable disorder is present in the case of Ref. [23], and our predictions are not directly testable at present in this kind of structures. A clean quantum wire with a single occupied transverse channel represents a possible realization of a one-dimensional model that has been achieved using cleaved edge overgrowth [24] or by local oxidation [25] techniques in GaAs-GaAlAs heterostructures. Well defined conductance plateaus as a function of the gate voltage are obtained in Ref. [24], and the single-mode regime is reached. Gating a part of the wire will allow to vary locally the electron density leading to an increased importance of the interactions close to the gate and thus defining our nanosystem. However, the screening induced by the gate might weaken the increase of the effective electronelectron interaction arising from the low local density [26]. If the gate is not too close to the quantum wire, the importance of the interactions increases only gradually along the wire as we approach the nanosystem. Therefore the conductance of the nanosystem can be expected to be perfect, independent of the precise value of the interaction strength $[4,27,28]$. A nearby scanning gate microscope (SGM) would correspond to the one-body scatterer of our model.

A dependence of the total conductance of a perfectly transmitting nanosystem on the distance between the nanosystem and the tip of the SGM will be a clear consequence of the nonlocal interaction effect. In a quantum point contact close to pinch-off, strong values of the interaction strength up to $U \approx 2 \pi$ result from estimations of the screened on-site Coulomb interaction using a twodimensional setup [29]. This estimation, which is conservative for the one-dimensional case of interest falls in the range of interaction strengths where we observe large oscillations of the total conductance with the position of the tip (see Figs. 2, 3). For these values of the interaction strength, considering only mean-field and exchange effects becomes unreliable in our one-dimensional models, and more exact methods like DMRG are needed.

A complementary test in clean quantum wires would be to vary the gate voltage from close-to-open to close- 
to-pinch-off, thus changing the electron density and the strength of the effective interaction while remaining in a single-channel situation. In that case we expect to observe a gate-voltage dependence of the total conductance, that would be absent if the tip were removed.

The impressive advances of SGM allow to envision other tests of our model. Recently this experimental technique has been applied to more complicated setups, like the proximity of a quantum point contact [30], the imaging of a one-electron quantum dot in a nanowire [31], and Aharonov-Bohm rings [32]. In the latter experiments the imaging of wave functions inside open quantum rings was achieved. Numerical calculations neglecting interactions yield patterns resembling the observed ones. Such a correspondence might be explained by the fact that the rings are relatively large and contain many transverse channels, such that interaction effects might not be very important. Going to smaller structures and eventually to the singlechannel configuration will enhance the role of interactions, thus yielding a nanosystem in the sense of our interacting model region. We have seen that positioning a one-body scatterer inside the nanosystem (negative $L_{\mathrm{C}}$ in Figs. 2 and (3) is also a way of exploring electronic interactions. The conductance obtained when the tip is close or inside the nanosystem can be very different from the conductance resulting only from the backscattering by the tip.

In summary, we have demonstrated the importance of nonlocal interaction effects in quantum transport through nanostructures. These effects are particularly striking when the dressing cloud of a nanosystem with perfect transmission is perturbed by a one-body scatterer. We have suggested experimental setups in which the predicted effects can be detected.

$$
* * *
$$

We thank P. Schmitteckert for his DMRG code and useful discussions. Financial support has been provided by the European Union through the MCRTN program (contract MCRTN-CT-2003-504574).

\section{REFERENCES}

[1] Datta S., Electronic Transport in Mesoscopic Systems (Cambridge University Press, Cambridge) 1995.

[2] Sushrov O. P., Phys. Rev. B, 64 (2001) 155319.

[3] Molina R. A., Weinmann D. and Pichard J.-L., Europhys. Lett., 67 (2004) 96.

[4] Molina R. A., Weinmann D., Jalabert R. A., InGOld G.-L. and Pichard J.-L., Phys. Rev. B, 67 (2003) 235306.

[5] Goldhaber-Gordon D., Shtrikman H., Mahalu D., Abusch-Magder D., Meirav U. and Kastner M. A., Nature, 391 (1998) 156.

[6] Pustilnik M., Glazman L. I., Cobden D. H. and Kouwenhoven L. P., Lect. Notes Phys., 579 (2001) 3.

[7] Hewson A. C., The Kondo Problem to Heavy Fermions (Cambridge University Press, Cambridge) 1997.
[8] Bergmann G., Phys. Rev. B, 77 (104401) 2008.

[9] Asada Y., Freyn A. and Pichard J.-L., Eur. Phys. J. $B, 53$ (2006) 109.

[10] Freyn A. and Pichard J.-L., Phys. Rev. Lett., 98 (2007) 186401.

[11] Freyn A. and Pichard J.-L., Eur. Phys. J. B, 58 (2007) 279.

[12] Molina R. A., Weinmann D. and Pichard J.-L., Eur. Phys. J. B, 48 (2005) 243.

13] Topinka M. A., LeRoy B. J., Shaw S. E. J., Heller E. J., Westervelt R. M., Maranowski K. D. and Gossard A. C., Science, 289 (2000) 2323.

[14] Topinka M. A., LeRoy B. J., Westervelt R. M., Shaw S. E. J., Fleischmann R., Heller E. J., Maranowski K. D. and Gossard A. C., Nature, 410 (2001) 183

[15] Freyn A., Kleftogiannis I. and Pichard J.-L., arXiv:0801.3970v1.

[16] Molina R. A., Schmitteckert P., Weinmann D., Jalabert R. A., Ingold G.-L. and Pichard J.-L., Eur. Phys. J. B, 39 (2004) 107.

[17] Favand J. and Mila F., Eur. Phys. J B, 2 (1998) 293.

[18] Meden V. and Schollwöck U., Phys. Rev. B, 67 (2003) 193303.

[19] RejeC T. and RAmšAK A., Phys. Rev. B, 68 (2003) 035342 .

[20] Peschel I., Wang X., Kaulke M. and Hallberg K. (Editors), Density-Matrix Renormalization - A New Numerical Method in Physics (Springer, Berlin, Heidelberg) 1999.

[21] SCHMitTeCKeRT P., Interplay between interaction and disorder in one-dimensional Fermi systems, PhD Thesis, Universität Augsburg, 1996.

[22] Vasseur G., Weinmann D. and Jalabert R. A., Eur. Phys. J. B, 51 (2006) 267.

[23] Hofheinz M., Jehl X., Sanquer M., Molas G., Vinet M. and Deleonibus S., Appl. Phys. Lett., 89 (2006) 143504.

[24] Yacobi A., Stormer H. L., Wingreen N. S., Pfeiffer L. N., Baldwin K. W. and West K. W., Phys. Rev. Lett., 77 (1996) 4612.

[25] Kvon Z. D., Galaktionov E. A., Sablikov V. A., Savchenko A. K., ShCheglov D. A. and Latyshev A. V., JETP Lett., 83 (2006) 458.

[26] Aleiner I. L. and Shklovskit B. I., Phys. Rev. B, 49 (1994) 13721

[27] Maslov D. L. and Stone M., Phys. Rev. B, 52 (1995) R5539.

[28] Safi I. and Schulz H. J., Phys. Rev. B, 52 (1995) R17040.

[29] Lassl A., Schlagheck P. and Richter K., Phys. Rev. $B, 75$ (2007) 045346.

[30] Aoki N., Burke A., da Cunha C. R., Akis R., Ferry D. K. and Ochiai Y., J. Phys.: Conf. Ser., 38 (2006) 79.

[31] Bleszynski A. C., Fröberg L. E., Björk M. T., TroDAhl H. J., Samuelson L. and Westervelt R. M., arXiv:0802.3236v1.

[32] Martins F., Hackens B., Pala M. G., Ouisse T. Sellier H., Wallart X., Bollaert S., Cappy A., Chevrier J., Bayot V. and Huant S., Phys. Rev. Lett., 99 (2007) 136807. 